\title{
Peristaltic transport of a particle-fluid suspension through a uniform and non-uniform annulus
}

\author{
K.S. Mekheimer ${ }^{\mathrm{a}}$ and Y. Abd elmaboud ${ }^{\mathrm{b} *}$ \\ ${ }^{a}$ Mathematics Department, Al-Azhar University, Nasr City, Cairo, Egypt; ${ }^{b}$ Mathematics Department, Al-Azhar University \\ (Assiut Branch), Assiut, Egypt.
}

(Received 23 June 2008; final version received 27 July 2008)

\begin{abstract}
This study looks at the influence of an endoscope on the peristaltic flow of a particle-fluid suspension (as blood model) through tubes. A long wavelength approximation through a uniform and non-uniform infinite annulus filled with an incompressible viscous and Newtonian fluid mixed with rigid spherical particles of identical size is investigated theoretically. The inner tube is uniform, rigid and moving with a constant velocity $V_{0}$, whereas the outer non-uniform tube has a sinusoidal wave travelling down its wall. The axial velocity of the fluid phase $u_{\mathrm{f}}$, particulate phase $u_{\mathrm{p}}$ and the pressure gradients have been obtained in terms of the dimensionless flow rate $\bar{Q}$, the amplitude ratio $\phi$, particle concentration $C$, the velocity constant $V_{0}$ and the radius ratio $\epsilon$ (the ratio between the radius of the inner tube and the radius of the outer one at the inlet). Numerical calculations for various values of the physical parameters of interest are carried out for the pressure rise and the friction force on the inner and the outer tubes.
\end{abstract}

Keywords: peristaltic transport; particle-fluid suspension; non-uniform tube; pressure rise

\section{Introduction}

Peristaltic pumping is a form of fluid transport that occurs when a progressive wave of area contraction or expansion propagates along the length of distensible duct containing liquid or mixture. Peristalsis is now well known to physiologists as one of the major mechanisms for fluid transport in many biological systems. Certain physiological phenomena like urine transport from kidney to bladder through the ureter, movement of chyme in the gastrointestinal tract, the movements of spermatozoa in the ductus efferentes of the male reproductive tract and the ovum in the female fallopian tube, the locomotion of some worms and the vasomotion of small blood vessels involve peristaltic motion. In addition, peristaltic pumping occurs in many practical applications involving biomechanical systems such as roller and finger pumps. The effect of an endoscope on peristaltic motion of a fluid with suspended particles is very important for medical diagnosis, and it has many clinical applications. The endoscope is now a very important tool used for determining real reasons responsible for many problems in human organs in which the fluids are transported by peristaltic pumping such as, stomach, small intestine (Hayat et al. 2006; Mekheimer and Abd elmaboud 2008), etc. Also, from a fluid dynamic point of view, there is no difference between an endoscope and a catheter. Furthermore, the insertion of a catheter in an artery will alter the flow field and modify the pressure distribution. In addition, physio- logical organs are generally observed to be a non-uniform duct (Wiedeman 1963; Lee and Fung 1971). Hence, peristaltic analysis of a fluid in a uniform geometry cannot be applied when explaining the mechanism of fluid transport in most biosystems. Moreover, it is known that urine from the kidneys passes through ureters and is trapped within the contracted segments of the ureter. When there is some obstruction in the ureter or in the ureter-bladder junction, the upstream ureter dilates. In such hydroureter cases, peristaltic motion becomes a travelling wave of relatively small amplitude over a cylindrical tapered tube. It is evident that the efficiency of pumping is decreased in such cases and the quantity of urine pumped through the ureter is reduced. Ultimately, the urine is stored in the bladder, from which it is periodically delivered through the urethra (Misra and Pandey 1994).

A number of analytical (Shapiro et al. 1969; Zien and Ostrach 1970; El Shehawey and Mekheimer 1994; Ramachandra and Usha 1995; Mekheimer et al. 1998; Mekheimer 2002, 2003a, 2003b; Vajravelu et al. 2005; Hakeem et al. 2006), numerical and experimental (Latham 1966; Brown and Hung 1977; Takabatake and Ayukawa 1982, 1988; Tang and Shen 1993; Ratish Kumar and Naidu 1995) studies of peristaltic flows of different fluids have been reported. Recently, there have been some studies of peristaltic flows of non-Newtonian fluids (Hayat, Wang et al. 2002; Hayat, Mohomed et al. 2005; Hayat and Ali

*Corresponding author. E-mail: yass_math@yahoo.com 
2006; Hayat et al. 2006; Ali et al. 2007; Hayat, Afsar et al. 2007; Hayat, Ali, Abbas 2007; Hayat, Ali et al. 2007a, 2007b, 2007c; Ali et al. 2008; Wang et al. 2008). The study of the flow of mixtures is very useful in understanding diverse physical problems in many areas of technical importance including powder technology, fluidisation, sedimentation, combustion, aerosol fiteration, atmospheric fallout, lunar ash flows, etc. The continuum theory of mixtures is also applicable to hydrodynamics of biological systems, because it provides an improved understanding of diverse subjects such as diffusion of proteins, the rheology of blood, swimming of microorganisms and particle deposition in the respiratory tract (Srivastava and Srivastava 1989, 1997; Misra and Pandey 1994; Srivastava and Saxena 1997), etc.

Several solutions with various degrees of approximation are available in the literature for the peristaltic flow with various considerations of the nature of the fluid and the geometry of the tube (Misra and Pandey 1995; Eytan et al. 2001; Elshehawey et al. 2005). This study is essentially an extension of these works. The aim of this study is understanding the peristaltic transport of a particle-fluid mixture through the gap between coaxial tubes, where the outer tube is non-uniform and has a sinusoidal wave travelling down its wall and the inner one is a rigid, uniform tube, moving with a constant velocity, $V_{0}$. A motivation of the present analysis is the hope that such a problem will be applicable in many clinical applications such as the endoscope problem.

\section{Formulation of the problem}

Consider two coaxial infinite tubes, the gap between them filled with a mixture of small spherical rigid particles in an incompressible Newtonian viscous fluid - the outer tube is non-uniform and has a sinusoidal wave travelling down its wall and the inner one is a rigid, uniform tube and moving with a constant velocity. The geometry of the walls' surface is described as

$$
\begin{aligned}
& r_{1}^{\prime}=a_{1}, \\
& r_{2}^{\prime}=a_{2}\left(z^{\prime}\right)+b \sin \left(\frac{2 \pi}{\lambda}\left(z^{\prime}-c t^{\prime}\right)\right),
\end{aligned}
$$

with

$$
a_{2}\left(z^{\prime}\right)=a_{20}+k z^{\prime},
$$

where $a_{1}$ is the radius of the inner tube, $a_{2}\left(z^{\prime}\right)$ is the radius of the outer tube at any axial distance $z^{\prime}$ from the inlet, $a_{20}$ is the radius of the outer tube at the inlet (constant), $k(\ll 1)$ is a constant whose magnitude depends on the length of the outer tube, $b$ is the wave amplitude, $\lambda$ is the wavelength, $c$ is the propagation velocity, and $t^{\prime}$ is the time.

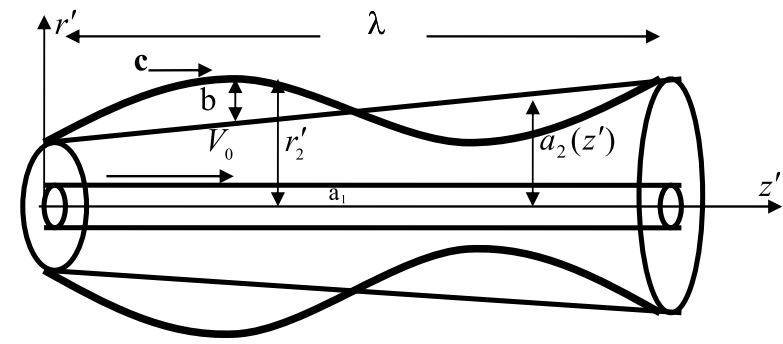

Figure 1. Geometry of the problem.

Using the cylindrical coordinate system $\left(r^{\prime}, z^{\prime}\right)$, where the $z^{\prime}$ axis lies along the centreline of the inner and the outer tubes, and $r^{\prime}$ is the distance measured radially. With the continuum mechanics approach, the equations governing the conservation of mass and linear momentum for both the fluid and the solid particle phases (Drew 1979; Misra and Pandey 1994) neglecting the inertia terms may be written in the following manner: Fluid phase

$$
\begin{aligned}
(1-C) \frac{\partial p^{\prime}}{\partial r^{\prime}}= & (1-C) \mu_{\mathrm{s}}(C)\left[\frac{\partial^{2}}{\partial r^{\prime 2}}+\frac{\partial^{2}}{\partial z^{\prime 2}}+\frac{1}{r^{\prime}} \frac{\partial}{\partial r^{\prime}}\right. \\
& \left.-\frac{1}{r^{\prime 2}}\right] v_{\mathrm{f}}^{\prime}+C S\left(v_{\mathrm{p}}^{\prime}-v_{\mathrm{f}}^{\prime}\right), \\
(1-C) \frac{\partial p^{\prime}}{\partial z^{\prime}}= & (1-C) \mu_{\mathrm{s}}(C)\left[\frac{\partial^{2}}{\partial r^{\prime 2}}+\frac{\partial^{2}}{\partial z^{\prime 2}}+\frac{1}{r^{\prime}} \frac{\partial}{\partial r^{\prime}}\right] u_{\mathrm{f}}^{\prime} \\
& +C S\left(u_{\mathrm{p}}^{\prime}-u_{\mathrm{f}}^{\prime}\right), \\
\frac{\partial}{\partial r^{\prime}}(1-C) v_{\mathrm{f}}^{\prime}+ & \frac{\partial}{\partial z^{\prime}}(1-C) u_{\mathrm{f}}^{\prime}+\frac{1}{r^{\prime}}(1-C) v_{\mathrm{f}}^{\prime}=0 .
\end{aligned}
$$

Particulate phase

$$
\begin{aligned}
& C \frac{\partial p^{\prime}}{\partial r^{\prime}}=C S\left(v_{\mathrm{f}}^{\prime}-v_{\mathrm{p}}^{\prime}\right), \\
& C \frac{\partial p^{\prime}}{\partial z^{\prime}}=C S\left(u_{\mathrm{f}}^{\prime}-u_{\mathrm{p}}^{\prime}\right), \\
& \frac{\partial}{\partial r^{\prime}} C v_{\mathrm{p}}^{\prime}+\frac{\partial}{\partial z^{\prime}} C u_{\mathrm{p}}^{\prime}+\frac{1}{r^{\prime}} C v_{\mathrm{p}}^{\prime}=0 .
\end{aligned}
$$

Where $u_{\mathrm{f}}^{\prime}$ and $v_{\mathrm{f}}^{\prime}$ are the axial and radial velocity components of the fluid phase, and $u_{\mathrm{p}}^{\prime}$ and $v_{\mathrm{p}}^{\prime}$ those of the particulate phase, $p^{\prime}$ is the pressure, $C$ is the constant (Srivastava and Saxena 1997) volume fraction density of the particles, $\mu_{\mathrm{s}}(C)$ is the mixture viscosity (effective or apparent viscosity of suspension), and $S$ is the drag coefficient of interaction for the force exerted by one phase on the other. The expression for the drag coefficient of interaction, $S$, and the empirical relation for the viscosity of the suspension, $\mu_{s}$, for the present problem is selected as in Srivastava and 
Saxena (1997),

$$
\begin{gathered}
S=\frac{9}{2} \frac{\mu_{0}}{a_{0}^{2}} \lambda^{\prime}(C), \\
\lambda^{\prime}(C)=\frac{4+\left[8 C-3 C^{2}\right]^{\frac{1}{2}}+3 C}{(2-3 C)^{2}},
\end{gathered}
$$

and

$$
\begin{gathered}
\mu_{\mathrm{s}}=\mu_{\mathrm{s}}(C)=\frac{\mu_{0}}{1-m C}, \\
m=0.070 \exp \left[2.49 C+\frac{1107}{T} \exp (-1.69 C)\right],
\end{gathered}
$$

where $a_{0}$ is the radius of each solid particle suspended in the fluid, $\mu_{0}$ is the constant fluid viscosity, and $T$ is absolute temperature measured in ${ }^{\circ} \mathrm{K}$. Formula (7) has been tested by Charm and Kurland (1974) by using a cone and a plate viscometer, and it has been proclaimed that it is reasonably accurate up to $C=0.6$.

We introduce the following nondimensional variables

$$
\begin{aligned}
r= & \frac{r^{\prime}}{a_{20}}, z=\frac{z^{\prime}}{\lambda}, u_{\mathrm{f}}=\frac{u_{\mathrm{f}}^{\prime}}{c}, u_{\mathrm{p}}=\frac{u_{\mathrm{p}}^{\prime}}{c}, v_{\mathrm{f}}=\frac{\lambda v_{\mathrm{f}}^{\prime}}{a_{20} c} \\
v_{\mathrm{p}}= & \frac{\lambda \mathrm{v}_{\mathrm{p}}^{\prime}}{a_{20} c}, t=\frac{c t^{\prime}}{\lambda}, p=\frac{a_{2}^{20}}{\lambda \mu_{0} c} p^{\prime}, \delta=\frac{a_{20}}{\lambda}, \bar{\mu}=\frac{\mu_{\mathrm{s}}}{\mu_{0}} \\
V_{0}= & \frac{V_{0}^{\prime}}{c}, r_{1}=\frac{r_{1}^{\prime}}{a_{20}}=\epsilon, r_{2}=\frac{r_{2}^{\prime}}{a_{20}}=1+\frac{\lambda k z}{a_{20}} \\
& +\phi \sin \left(\frac{2 \pi}{\lambda}(z-t)\right),
\end{aligned}
$$

where $\phi$ (amplitude ratio) $=\frac{b}{a_{20}}<1$ and $\delta$ is wave number ratio. To proceed, we non-dimensionalise Equations (3-8); this yields

Fluid phase

$$
\begin{gathered}
\frac{\partial p}{\partial r}=\bar{\mu} \delta^{2}\left[\frac{\partial^{2}}{\partial r^{2}}+\delta^{2} \frac{\partial^{2}}{\partial z^{2}}+\frac{1}{r} \frac{\partial}{\partial r}-\frac{1}{r^{2}}\right] v_{\mathrm{f}} \\
+\frac{C S a_{2}^{20}}{\mu_{0}(1-C)} \delta^{2}\left(v_{\mathrm{p}}-v_{\mathrm{f}}\right), \\
\frac{\partial p}{\partial z}=\bar{\mu}\left[\frac{\partial^{2}}{\partial r^{2}}+\delta^{2} \frac{\partial^{2}}{\partial z^{2}}+\frac{1}{r} \frac{\partial}{\partial r}\right] u_{\mathrm{f}} \\
+\frac{C S a_{2}^{20}}{\mu_{0}(1-C)}\left(u_{\mathrm{p}}-u_{\mathrm{f}}\right),
\end{gathered}
$$

$$
\frac{\partial}{\partial r}(1-C) v_{\mathrm{f}}+\frac{\partial}{\partial z}(1-C) u_{\mathrm{f}}+\frac{1}{r}(1-C) v_{\mathrm{f}}=0 .
$$

Particulate phase

$$
\begin{aligned}
& \frac{\partial p}{\partial r}=\frac{S a_{2}^{20}}{\mu_{0}} \delta^{2}\left(v_{f}-v_{\mathrm{p}}\right), \\
& \frac{\partial p}{\partial z}=\frac{S a_{2}^{20}}{\mu_{0}}\left(u_{\mathrm{f}}-u_{\mathrm{p}}\right), \\
& \frac{\partial}{\partial r} C v_{\mathrm{p}}+\frac{\partial}{\partial z} C u_{\mathrm{p}}+\frac{1}{r} C v_{\mathrm{p}}=0 .
\end{aligned}
$$

Using the long wavelength approximation of (Shapiro and Jaffrin 1969; Mekheimer 2008; Mekheimer and Abd elmaboud 2008a, 2008c), the appropriate equations of momentum for both the fluid and the particle phase are (Srivastava and Saxena 1997)

$$
\begin{aligned}
& \frac{\partial p}{\partial r}=0, \\
& \frac{\partial p}{\partial z}=\frac{\bar{\mu}}{r} \frac{\partial}{\partial r}\left(r \frac{\partial}{\partial r}\right) u_{\mathrm{f}}+\frac{C S a_{2}^{20}}{(1-C) \mu_{0}}\left(u_{\mathrm{p}}-u_{\mathrm{f}}\right), \\
& \frac{\partial p}{\partial z}=\frac{S a_{2}^{20}}{\mu_{0}}\left(u_{\mathrm{f}}-u_{\mathrm{p}}\right) .
\end{aligned}
$$

Equation (18) shows that $p$ is not a function of $r$. The only important contribution of the non-dimensional boundary conditions is

$$
\begin{gathered}
u_{\mathrm{f}}=V_{0} \quad \text { at } \quad r=r_{1}=\epsilon, \quad \text { and } \quad u_{\mathrm{f}}=0 \\
\text { at } \quad r=r_{2}=1+\frac{\lambda k z}{a_{20}}+\phi \sin 2 \pi(z-t) .
\end{gathered}
$$

The expressions for the velocity profiles of the fluid phase, $u_{\mathrm{f}}$, and the particle phase, $u_{\mathrm{p}}$, obtained as the solutions of Equations (19) and (20) subject to the boundary conditions (21) are given as

$$
\begin{aligned}
u_{\mathrm{f}}(r, z, t)= & \frac{-1}{4(1-C) \bar{\mu}} \frac{d p}{d z}\left\{\left(r_{2}^{2}-r_{1}^{2}\right)\left(\frac{\ln \left(\frac{r}{r_{1}}\right)}{\ln \left(\frac{r_{2}}{r_{1}}\right)}\right)-r^{2}\right. \\
& \left.+r_{1}^{2}\right\}-\frac{V_{0} \ln \left(\frac{r}{r_{2}}\right)}{\ln \left(\frac{r_{2}}{r_{1}}\right)}, \\
u_{\mathrm{p}}(r, z, t)= & \frac{-1}{4(1-C) \bar{\mu}} \frac{d p}{d z}\left\{\left(r_{2}^{2}-r_{1}^{2}\right)\left(\frac{\ln \left(\frac{r}{r_{1}}\right)}{\ln \left(\frac{r_{2}}{r_{1}}\right)}\right)-r^{2}\right. \\
& \left.+r_{1}^{2}+\frac{4(1-C) \mu_{\mathrm{s}}}{a_{2}^{20} S}\right\}-\frac{V_{0} \ln \left(\frac{r}{r_{2}}\right)}{\ln \left(\frac{r_{2}}{r_{1}}\right)} .
\end{aligned}
$$


The instantaneous volume flow rate $Q(z, t)$ is given by

$$
\begin{aligned}
& Q(z, t)=Q_{\mathrm{f}}(z, t)+Q_{\mathrm{p}}(z, t), \\
& Q(z, t)= \frac{-\pi}{8 \bar{\mu}(1-C)} \frac{d p}{d z}\left\{( r _ { 2 } ^ { 2 } - r _ { 1 } ^ { 2 } ) \left(r_{2}^{2}+r_{1}^{2}\right.\right. \\
&\left.\left.-\left(\frac{r_{1}^{2}-r_{2}^{2}}{\ln \left(\frac{r_{2}}{r_{1}}\right)}\right)\right)+\beta\left(r_{2}^{2}-r_{1}^{2}\right)\right\} \\
&-\pi V_{0}\left(r_{1}^{2}+\frac{r_{1}^{2}-r_{2}^{2}}{2 \ln \left(\frac{r_{2}}{r_{1}}\right)}\right)
\end{aligned}
$$

where

$$
\begin{aligned}
& Q_{\mathrm{f}}(z, t)=2 \pi(1-C) \int_{r_{1}}^{r_{2}} r u_{\mathrm{f}} d r \\
& Q_{\mathrm{p}}(z, t)=2 \pi C \int_{r_{1}}^{r_{2}} r u_{\mathrm{p}} d r, \quad \beta=\frac{8 C(1-C) \mu_{\mathrm{s}}}{a_{2}^{20} S},
\end{aligned}
$$

with $\beta$ a non-dimensional suspension parameter. From Equation (24), we get

$$
\begin{aligned}
& \frac{d p}{d z}= \\
& \frac{-8 \bar{\mu}(1-C)\left[\left(Q \frac{(z, t)}{\pi}\right) \ln \left(\frac{r_{2}}{r_{1}}\right)+\frac{V_{0}}{2}\left(r_{1}^{2}-r_{2}^{2}\right)+V_{0} r_{1}^{2} \ln \left(\frac{r_{2}}{r_{1}}\right)\right]}{\left(r_{2}^{4}-r_{1}^{4}\right) \ln \left(\frac{r_{2}}{r_{1}}\right)-\left(r_{1}^{2}-r_{2}^{2}\right)^{2}+\beta\left(r_{2}^{2}-r_{1}^{2}\right) \ln \left(\frac{r_{2}}{r_{1}}\right)} .
\end{aligned}
$$

The pressure rise $\Delta p_{L}(t)$ and the friction force (at the wall) on the outer and inner tubes, $F_{L}^{(\mathrm{o})}(\mathrm{t})$ and $F_{L}^{(\mathrm{i})}(t)$, respectively, in a tube of length $L$, in their non-dimensional forms, are given by

$$
\begin{aligned}
\Delta p_{L}(t) & =\int_{0}^{\Lambda} \frac{d p}{d z} d z \\
F_{L}^{(0)}(t) & =\int_{0}^{\Lambda} r_{2}^{2}\left(-\frac{d p}{d z}\right) d z \\
F_{L}^{(i)}(t) & =\int_{0}^{\Lambda} r_{1}^{2}\left(-\frac{d p}{d z}\right) d z
\end{aligned}
$$

where $\Lambda=\frac{L}{\lambda}$.

Substituting from Equation (25) in Equations (26-28) and with $r_{1}=\epsilon$ and $r_{2}(z, t)=1+\frac{\lambda k z}{a_{20}}+\phi \sin 2 \pi(z-t)$, we get

$$
\begin{aligned}
\Delta p_{L}(t)= & -8 \bar{\mu}(1-C) \int_{0}^{\Lambda}\left\{\left(Q \frac{(z, t)}{\pi)}\right.\right. \\
& \times \ln \left(\frac{1+\frac{\lambda k z}{a_{20}}+\phi \sin 2 \pi(z-t)}{\epsilon}\right)
\end{aligned}
$$

$$
\begin{aligned}
& +\frac{V_{0}}{2}\left(\epsilon^{2}-\left(1+\frac{\lambda k z}{a_{20}}+\phi \sin 2 \pi(z-t)\right)^{2}\right) \\
& \left.+V_{0} \epsilon^{2} \ln \left(\frac{1+\frac{\lambda k z}{a_{20}}+\phi \sin 2 \pi(z-t)}{\epsilon}\right)\right\} \\
& \times\left\{1 /\left(\left(1+\frac{\lambda k z}{a_{20}}+\phi \sin 2 \pi(z-t)\right)^{4}-\epsilon^{4}\right)\right. \\
& \times \ln \left(\frac{1+\frac{\lambda k z}{a_{20}}+\phi \sin 2 \pi(z-t)}{\epsilon}\right)
\end{aligned}
$$$$
\begin{aligned}
& -\left(\epsilon^{2}-\left(1+\frac{\lambda k z}{a_{20}}+\phi \sin 2 \pi(z-t)\right)^{2}\right)^{2} \\
& +\beta\left(\left(1+\frac{\lambda k z}{a_{20}}+\phi \sin 2 \pi(z-t)\right)^{2}-\epsilon^{2}\right)
\end{aligned}
$$$$
\left.\ln \left(\frac{1+\frac{\lambda k z}{a_{20}}+\phi \sin 2 \pi(z-t)}{\epsilon}\right)\right\} d z
$$

$$
\begin{aligned}
& F_{L}^{(\mathrm{o})}(t)=8 \bar{\mu}(1-C) \\
& \times \int_{0}^{\Lambda}\left\{\left(1+\frac{\lambda k z}{a_{20}}+\phi \sin 2 \pi(z-t)\right)^{2}\right\} \\
& \times\left\{\left(\frac{Q(z, t)}{\pi} \ln \left[\frac{1+\frac{\lambda k z}{a_{20}}+\phi \sin 2 \pi(z-t)}{\epsilon}\right]\right.\right. \\
& +\frac{V_{0}}{2}\left[\epsilon^{2}-\left(1+\frac{\lambda k z}{a_{20}}+\phi \sin 2 \pi(z-t)\right)^{2}\right] \\
& \left.+V_{0} \epsilon^{2} \ln \left[\frac{1+\frac{\lambda k z}{a_{20}}+\phi \sin 2 \pi(z-t)}{\epsilon}\right]\right\} \\
& \times\left\{1 /\left(\left(1+\frac{\lambda k z}{a_{20}}+\phi \sin 2 \pi(z-t)\right)^{4}-\epsilon^{4}\right)\right. \\
& \times \ln \left(\frac{1+\frac{\lambda k z}{a_{20}}+\phi \sin 2 \pi(z-t)}{\epsilon}\right) \\
& -\left(\epsilon^{2}-\left(1+\frac{\lambda k z}{a_{20}}+\phi \sin 2 \pi(z-t)\right)^{2}\right)^{2} \\
& +\beta\left(\left(1+\frac{\lambda k z}{a_{20}}+\phi \sin 2 \pi(z-t)\right)^{2}-\epsilon^{2}\right) \\
& \left.\times \ln \left[\frac{1+\frac{\lambda k z}{a_{20}}+\phi \sin 2 \pi(z-t)}{\epsilon}\right)\right\} d z,
\end{aligned}
$$


$F_{L}^{(i)}(t)=8 \bar{\mu}(1-C)$

$$
\begin{aligned}
& \times \int_{0}^{\Lambda} \epsilon^{2}\left\{\left(\frac{Q(z, t)}{\pi}\right)\right. \\
& \times \ln \left[\frac{1+\frac{\lambda k z}{a_{20}}+\phi \sin 2 \pi(z-t)}{\epsilon}\right] \\
& +\frac{V_{0}}{2}\left[\epsilon^{2}-\left(1+\frac{\lambda k z}{a_{20}}+\phi \sin 2 \pi(z-t)\right)^{2}\right] \\
& \left.+V_{0} \epsilon^{2} \ln \left[\frac{1+\frac{\lambda k z}{a_{20}}+\phi \sin 2 \pi(z-t)}{\epsilon}\right]\right\} \\
& \times\left\{1 /\left[\left(1+\frac{\lambda k z}{a_{20}}+\phi \sin 2 \pi(z-t)\right)^{4}-\epsilon^{4}\right)\right. \\
& \times \ln \left[\frac{1+\frac{\lambda k z}{a_{20}}+\phi \sin 2 \pi(z-t)}{\epsilon}\right] \\
& -\left[\epsilon^{2}-\left(1+\frac{\lambda k z}{a_{20}}+\phi \sin 2 \pi(z-t)\right)^{2}\right]^{2} \\
& +\beta\left(\left(1+\frac{\lambda k z}{a_{20}}+\phi \sin 2 \pi(z-t)\right)^{2}-\epsilon^{2}\right) \\
& \left.\times \ln \left[\frac{1+\frac{\lambda k z}{a_{20}}+\phi \sin 2 \pi(z-t)}{\epsilon}\right]\right] d z .
\end{aligned}
$$

In the absence of the particle phase (i.e. $C=0$ ), Equations (29-31) are the same as those obtained by Mekheimer (2005); the limit of Equations (22) and (25), as $r_{1}$ tends to be zero in the absence of the particle phase (i.e. $C=0$ ), gives the forms of the axial velocity and the pressure gradient for peristaltic flow in a non-uniform tube, (without endoscope, $\epsilon=0$ ): they will be

$$
u_{\mathrm{f}}(r, z, t)=\frac{-1}{4}\left(\frac{d p}{d z}\right)\left(r_{2}^{2}-r^{2}\right)
$$

and

$$
\frac{d p}{d z}=\frac{-8 Q(z, t)}{\pi r_{2}^{4}}
$$

Hence, the pressure rise and the outer friction force in this case, respectively, take the forms

$$
\Delta p_{L}(t)=-8 \int_{0}^{\Lambda} \frac{Q(z, t) / \pi}{\left(1+\frac{\lambda k z}{a_{20}}+\phi \sin 2 \pi(z-t)\right)^{4}} d z
$$

$$
F_{L}^{(\mathrm{o})}(t)=8 \int_{0}^{\Lambda} \frac{Q(z, t) / \pi}{\left(1+\frac{\lambda k z}{a_{20}}+\phi \sin 2 \pi(z-t)\right)^{2}} d z .
$$

Equations (34) and (35) are the same results as those obtained by Gupta and Seshadri (1976), Srivastava and Srivastava for (1988) a non-Newtonian fluid when the power law index $n=1$, and also those obtained by Srivastava et al. (1983) for a constant viscosity $(\delta=0)$. Furthermore, if $k=$ 0 in Equations (34) and (35), we can get expressions for the pressure rise and friction force in a uniform tube, and these correspond to the results obtained by Shapiro and Jaffrin (1969) The analytical interpretations of our analysis with other theories are difficult to make at this stage; the integrals involved in Equations (29-31) are very difficult to evaluate analytically and, thus, will be evaluated numerically.

\section{Numerical results and discussion}

In order to have an estimate of the quantitative effects of the various parameters involved in the results of the present analysis, computer codes are developed for the numerical evaluations of the analytical results obtained for $\Delta p_{L}(t)$ and $F_{L}^{(\mathrm{o})}(t)$ and $F_{L}^{(\mathrm{i})}(t)$ for parameters values (Gupta and Seshadri 1976; Srivastava and Srivastava 1988, 1997; Cotton and Williams 1990): $C=0-0.6, \epsilon=0.32,0.38$ and $0.44, a_{20}=1.25 \mathrm{~cm}, L=\lambda=8.01 \mathrm{~cm}, k=\frac{3 a_{20}}{\lambda}$. We shall assume the form of the instantaneous volume rate of the flow, $Q(z, t)$, periodic in $(z-t)$ as (Gupta and Seshadri

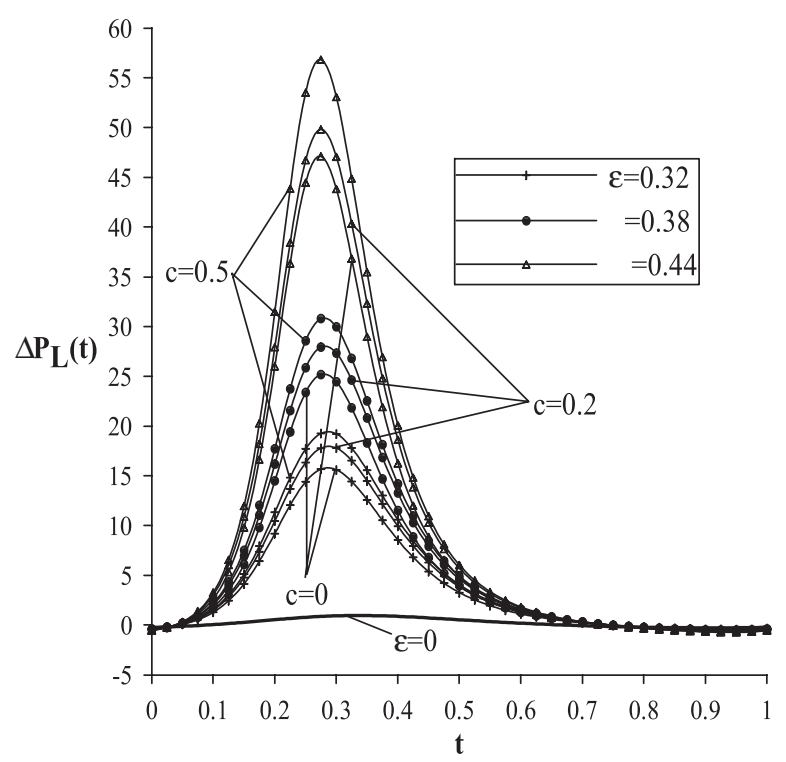

Figure 2. Variation of pressure rise over the length of a nonuniform annulus at $\phi=0.4$ and $V_{0}=0$, with different values of $\epsilon$ and $C$. 


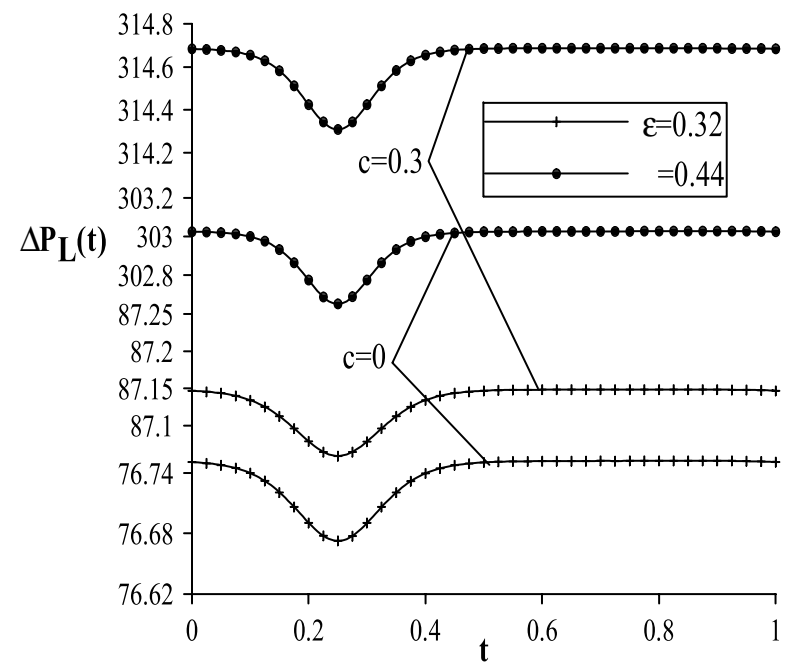

Figure 3. Variation of pressure rise over the length of a uniform annulus at $\phi=0.4$ and $V_{0}=0$, with different values of $\epsilon$ and $C$.

1976; Mekheimer 2005)

$$
\begin{aligned}
\frac{Q(z, t)}{\pi}= & \frac{\bar{Q}}{\pi}-\frac{\phi^{2}}{2}+2 \phi \sin 2 \pi(z-t)+\frac{2 \lambda k z}{a_{20}} \\
& \times \phi \sin 2 \pi(z-t)+\phi^{2} \sin ^{2} 2 \pi(z-t),
\end{aligned}
$$

where $\bar{Q}$ is the time average of the flow over one period of the wave. This form of $Q(z, t)$ has been assumed in view of the fact that the constant value of $Q(z, t)$ gives

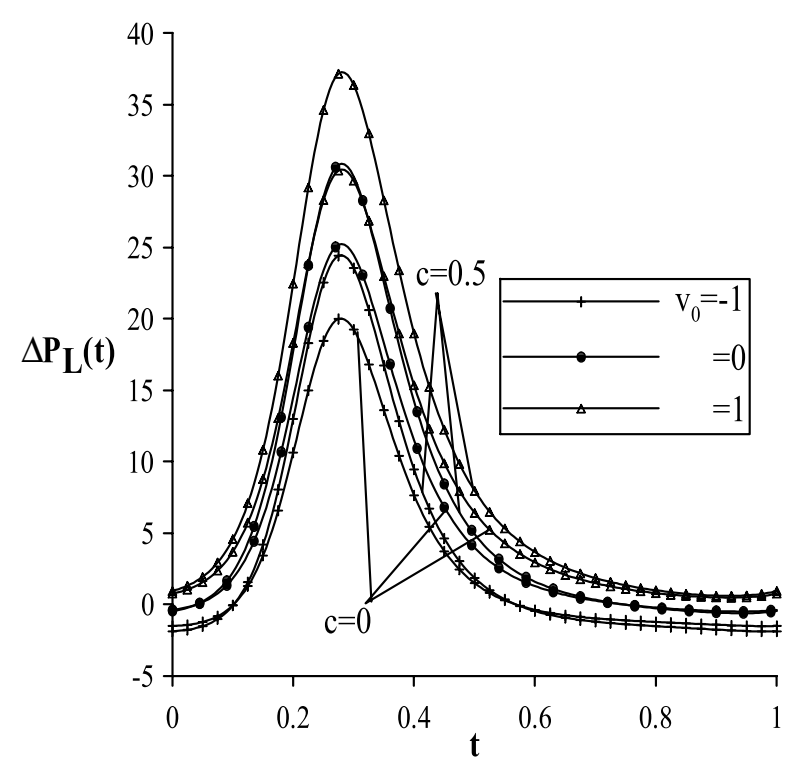

Figure 4. Variation of pressure rise over the length of a nonuniform annulus at $\phi=0.4$ and $\epsilon=0.38$, with different values of $V_{0}$ and $C$.

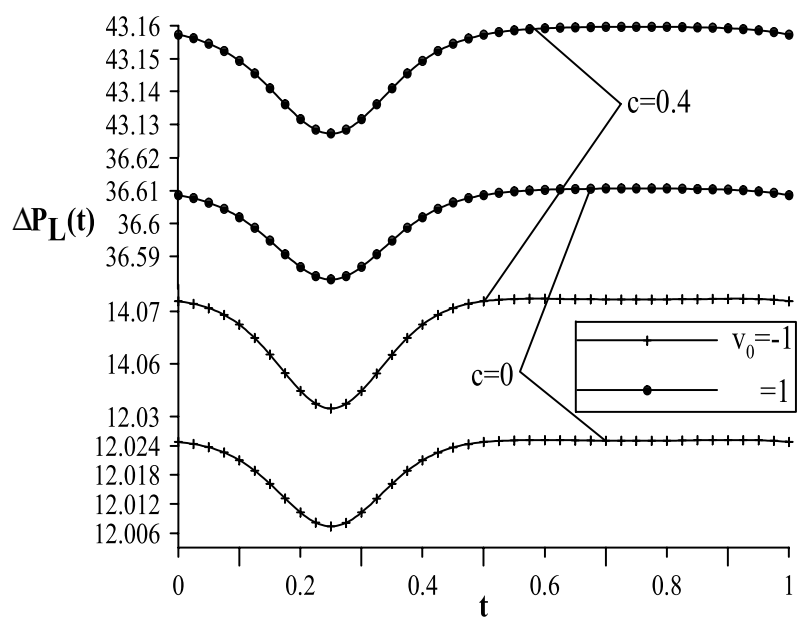

Figure 5. Variation of pressure rise over the length of a uniform annulus at $\phi=0.3$ and $\epsilon=0.32$, with different values of $V_{0}$ and $C$.

$\Delta p_{L}(t)$ always negative, and hence there will be no pumping action. We shall use the form of $Q(z, t)$ to compute the dimensionless pressure rise $\Delta p_{L}(t)$, the inner friction force $F_{L}^{(\mathrm{i})}(t)$ (on the inner surface) and the outer friction force $F_{L}^{(o)}(t)$ (on the outer tube) over the tube length for various values of the parameters. The average rise in pressure $\Delta \bar{p}_{L}$, outer friction force $\bar{F}_{L}^{(\text {o) }}$, and inner friction force $\bar{F}_{L}^{(\mathrm{i})}$ is then evaluated by averaging $\Delta p_{L}(t), F_{L}^{(o)}(t)$ and $F_{L}^{(\mathrm{i})}(t)$ over one period of the wave.

Figures (2) and (3) represent the variation of the dimensionless pressure rise with dimensionless time $t$ for $\phi=0.4$,

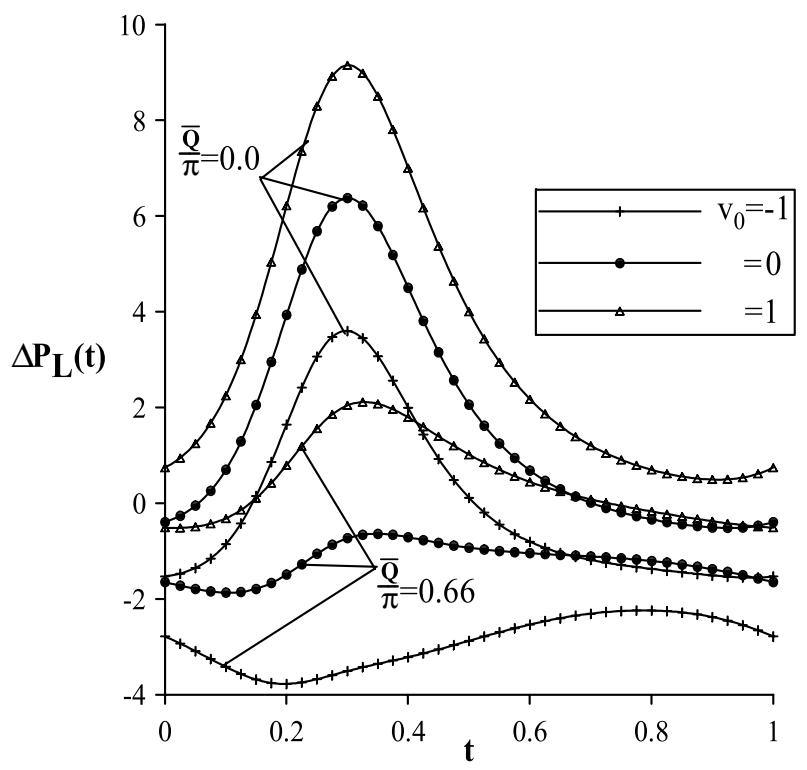

Figure 6. Variation of pressure rise over the length of a nonuniform annulus at $\phi=0.3, \epsilon=0.32$ and $C=0.1$, with different values of $\bar{Q}$ and $V_{0}$. 


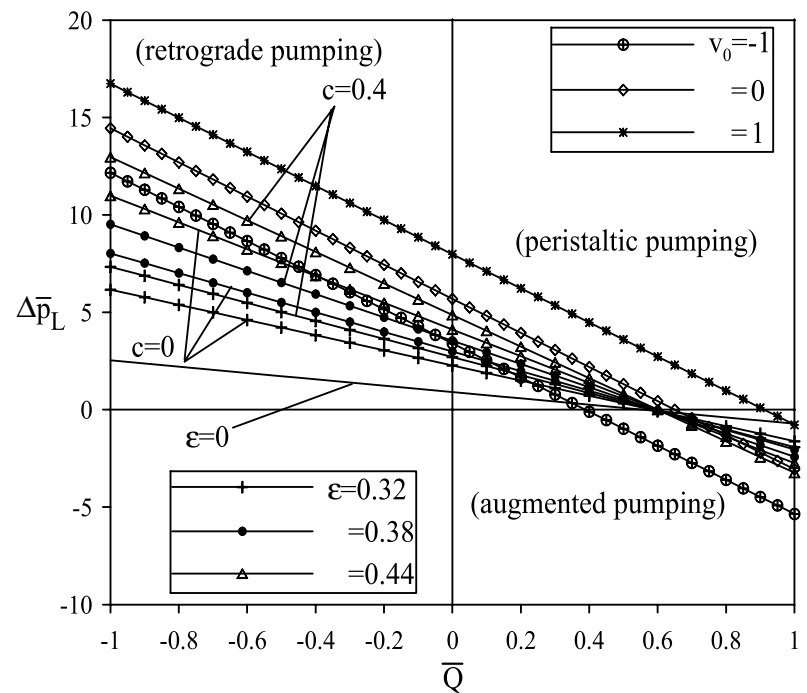

Figure 7. Pressure rise versus flow rate for a non-uniform annulus for different values of $\epsilon$ and $C$ at $\phi=0.3$ and $V_{0}=0.5$, and for different values of $V_{0}$ at $\phi=0.4, \epsilon=0.38$ and $C=0$.

$V_{0}=0$, with different values of $\epsilon$ and $C$ in the case of nonuniform and uniform tubes, respectively. It is evident that the pressure rise increases with the increase in the radius ratio, $\epsilon$, i.e. as the inner radius of the tube increases, and the pressure rise increases with increasing particle concentration, $C$. Figures 4 and 5 show that the variation of the dimensionless pressure rises with the dimensionless time $t$ for $\phi=0.4, \epsilon=0.38$, with different values of $V_{0}$ and $C$ for non-uniform tube and for $\phi=0.3, \epsilon=0.32$, with different values of $V_{0}$ and $C$ for uniform tube. The result shows that the pressure rise increases with the increase in the inner tube

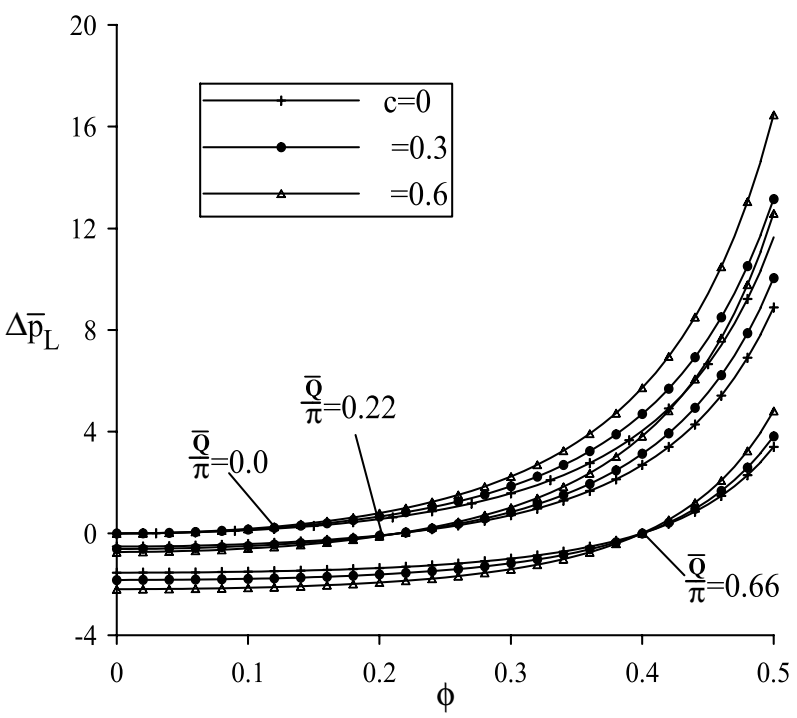

Figure 8. Pressure rise versus $\phi$ for a non-uniform annulus at different values of $\bar{Q}$ and $C$.

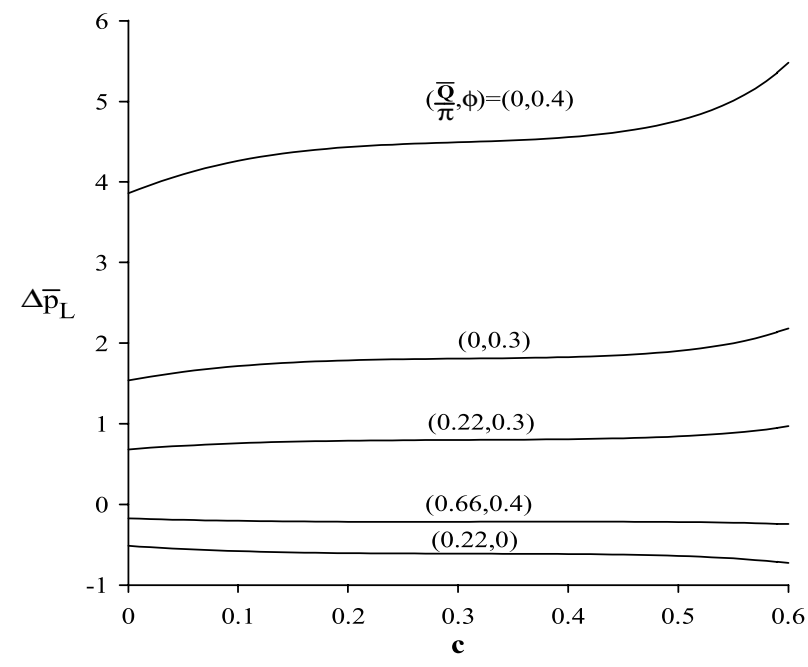

Figure 9. Pressure rise versus particle concentration, $C$, for a non-uniform annulus at different values of $\bar{Q}$ and $\phi$.

velocity i.e. the pressure rise for the endoscope increases as the inner tube moves in the direction of the peristaltic wave at the same particle concentration, $C$. The effect of the flow average, $\bar{Q}$, on the pressure rise profiles is depicted in Figure 6, it is clear that the effect of increasing the flow rate is to reduce the pressure rise for various values of $V_{0}$. Figures (2 and 3) further reveal that the magnitude of the pressure rise for a non-uniform tube is much smaller than the corresponding value for a uniform tube. This happens because the complete occlusion occurs only at the entry in a diverging tube, whereas in a uniform tube, it occurs at all points along the tube.

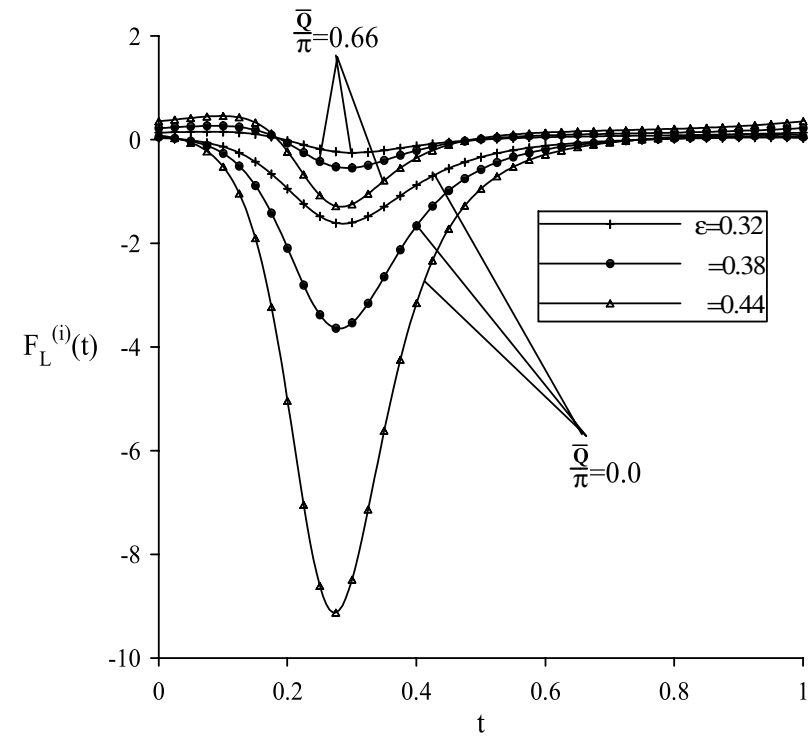

Figure 10. Variation of inner friction force over the length of a non-uniform annulus at $\phi=0.4, V_{0}=0$ and $C=0$, with different values of $\epsilon$ and $\bar{Q}$. 


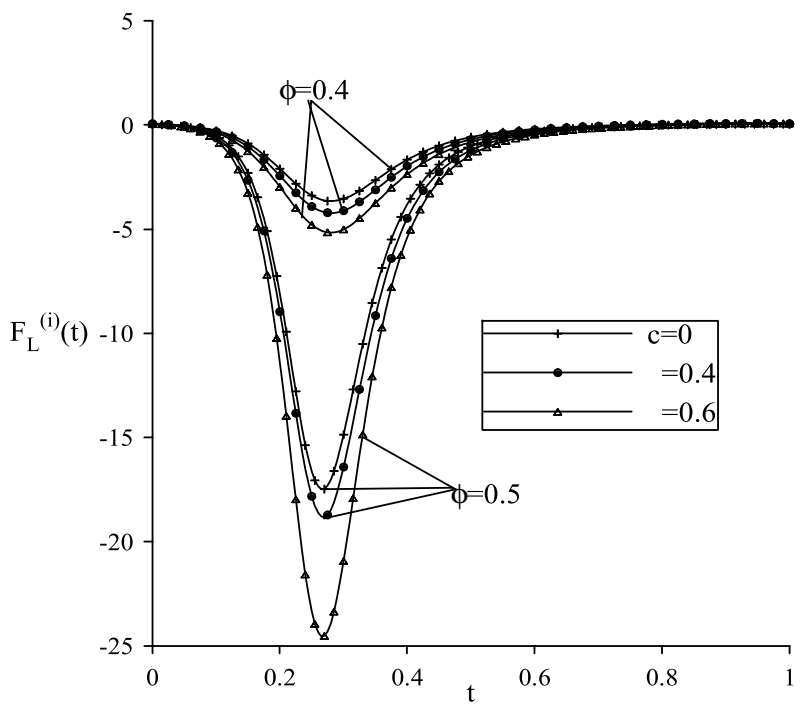

Figure 11. Variation of inner friction force over the length of a non-uniform annulus at $V_{0}=0$ and $\epsilon=0.38$ for different values of $C$ and $\phi$.

For a non-uniform tube, the average pressure rise is plotted versus the mean flow rate for different values of $C, V_{0}$ and $\epsilon$ in Figure 7; it is noticed that a linear relation between them and an increase in the flow rate reduce the pressure rise, and thus, the maximum flow rate is achieved at zero pressure rise, and maximum pressure occurs at zero flow rate. The effect of increasing $\epsilon$ and $C$ leads to increase in pressure rise. Finally, Figure 7 shows that the pressure rise increases with the increase in $V_{0}$ (the inner tube moves in the direction of the peristaltic wave).

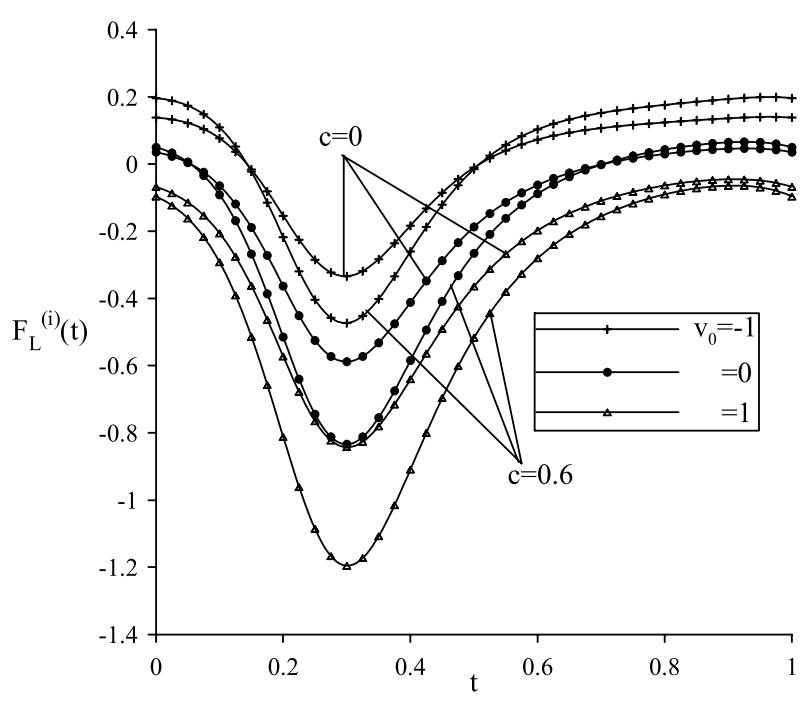

Figure 12. Variation of inner friction force over the length of a non-uniform annulus at $\phi=0.3$ and $\epsilon=0.38$ for different values of $C$ and $V_{0}$.

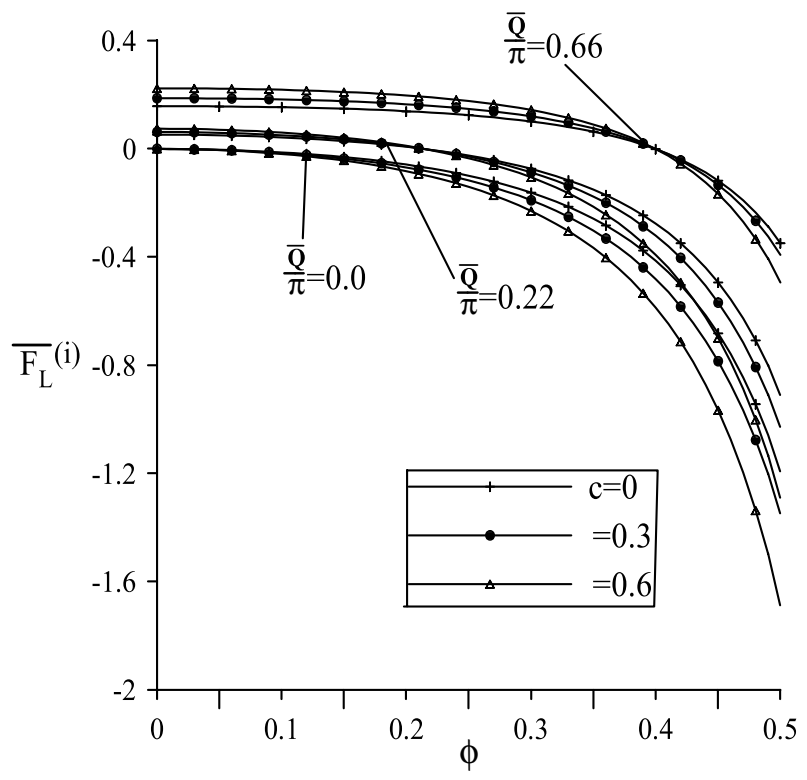

Figure 13. Inner friction force versus $\phi$ for a non-uniform annulus for different values of $\bar{Q}$ and $C$.

The pumping regions, peristaltic pumping $(\bar{Q}>0$ and $\left.\Delta \bar{p}_{L}>0\right)$, augmented pumping $\left(\bar{Q}>0\right.$ and $\left.\Delta \bar{p}_{L}<0\right)$ and retrograde pumping $\left(\bar{Q}<0\right.$ and $\left.\Delta \bar{p}_{L}>0\right)$ are also shown in Figure 7, and it is clear that the peristaltic pumping region becomes wider as the radius ratio, $\epsilon$, particle concentration $C$, and $V_{0}$ increase. A study of Figures 8 and 9 reveal that the pressure rise increases with the rise in the value of $\phi$ for

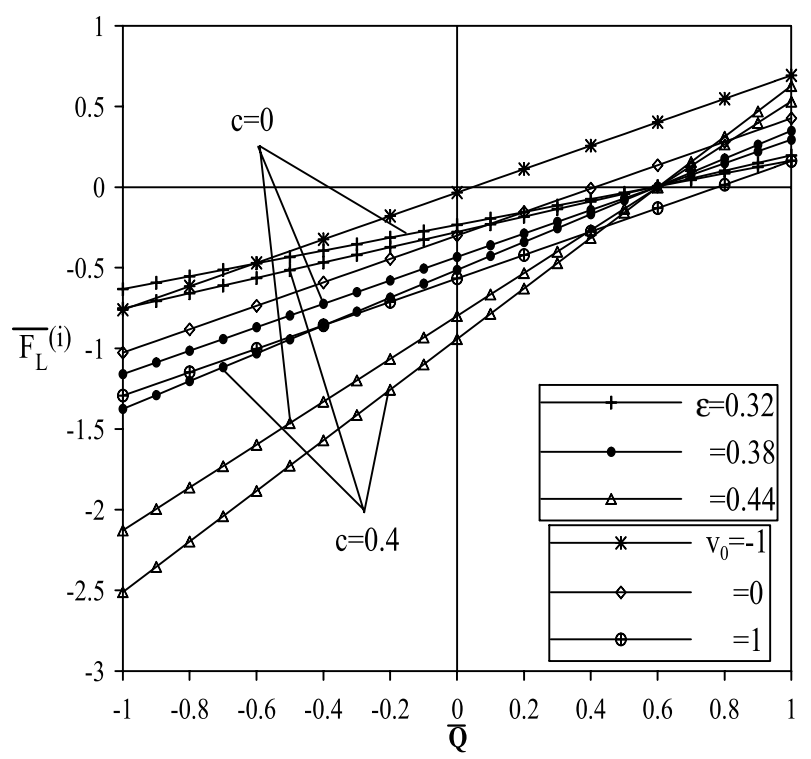

Figure 14. Inner friction force versus flow rate $\bar{Q}$ for a nonuniform annulus for different values of $\epsilon$ and $C$ at $\phi=0.3$ and $V_{0}=0.5$, and for different values of $V_{0}$ at $C=0, \phi=0.4$ and $\epsilon=0.38$. 


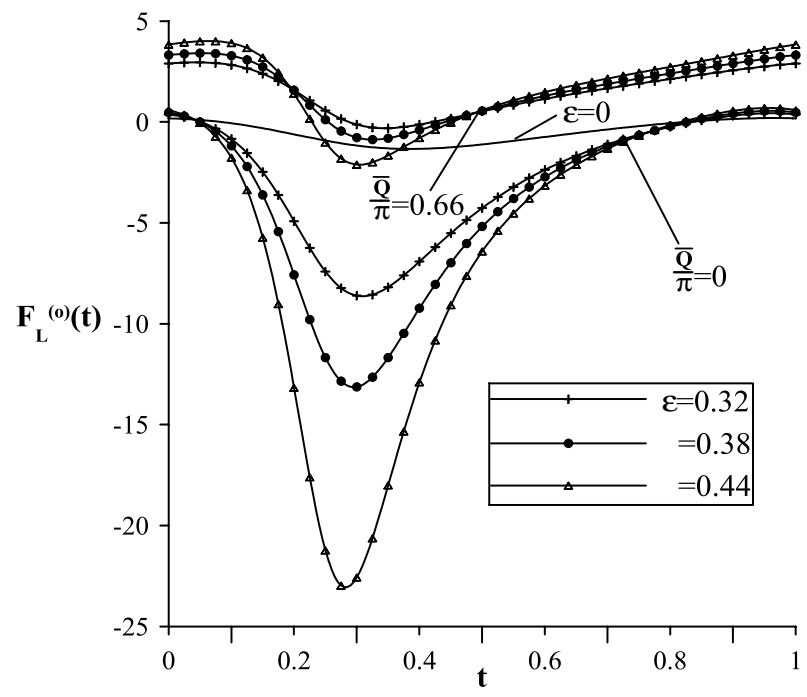

Figure 15. Variation of the outer friction force over the length of a non-uniform annulus at $\phi=0.4, V_{0}=0$ and $C=0$, with different values of $\epsilon$.

the other given fixed set of parameters. From Figure 9, we also notice that the pressure rise increases with increasing particle concentration, $C$.

Figures 10-14 describe the results obtained for the inner friction force versus the dimensionless time $t$ and the flow rate $\bar{Q}$, and Figures $15-19$ describe the results for outer friction force versus the dimensionless time $t$ and the flow rate $\bar{Q}$. Furthermore, the average outer friction forces in the case of an endoscope presence $(\epsilon \neq 0)$ and in the case of its absence $(\epsilon=0)$ are shown in Figure

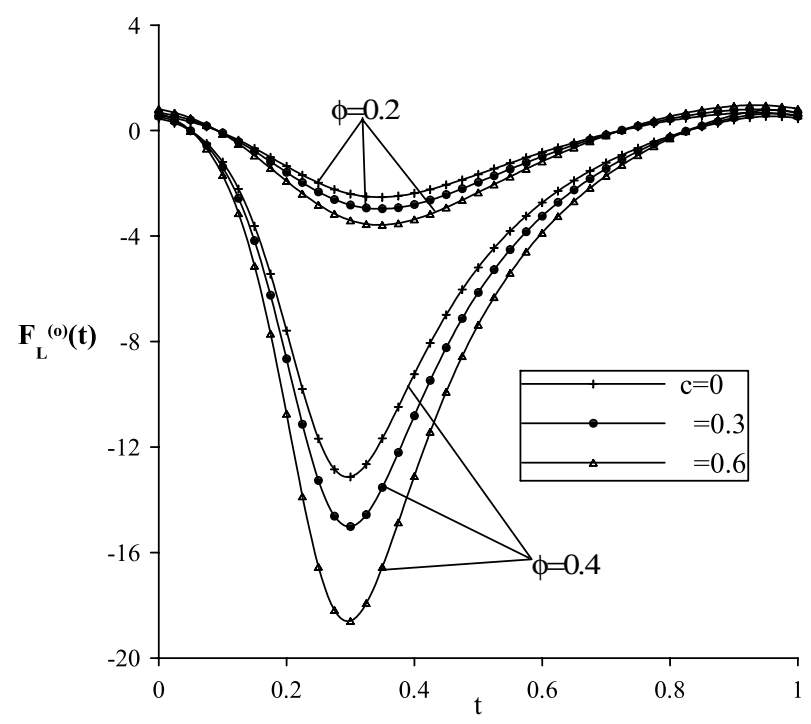

Figure 16. Variation of the outer friction force over the length of a non-uniform annulus at $V_{0}=0$ and $\epsilon=0.38$ for different values of $C$ and $\phi$.

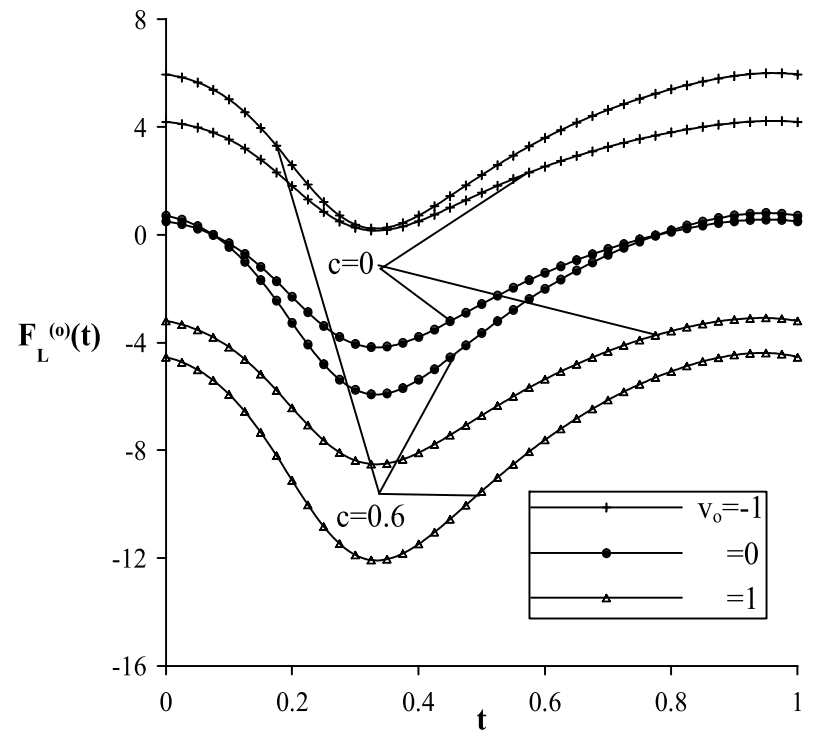

Figure 17. Variation of the outer friction force over the length of a non-uniform annulus at $\phi=0.3$ and $\epsilon=0.38$ for different values of $C$ and $V_{0}$.

19. The effect of important parameters such as $C, \epsilon, V_{0}$, $\phi$ and $\bar{Q}$ on the inner and outer friction force has been investigated.

We notice from these figures that the inner and outer friction forces have opposite behaviour compared with the pressure rise. The inner friction force behaves similar to the outer friction force for the same values of the parameters; moreover, the outer friction force is greater than the inner friction force at the same values of the parameters.

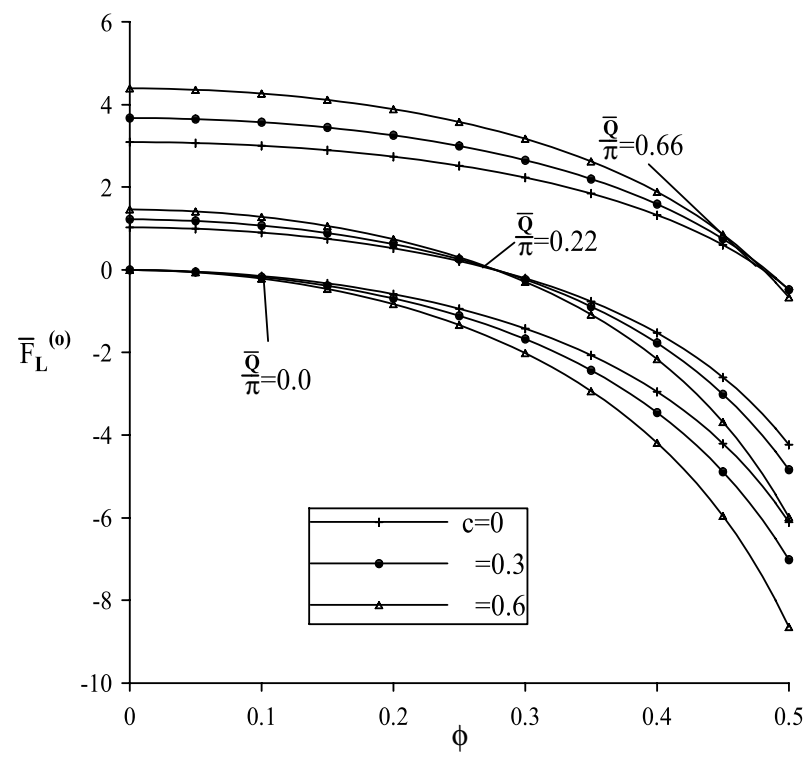

Figure 18. Outer friction force versus $\phi$ for a non-uniform annulus for different values of $\bar{Q}$ and $C$. 


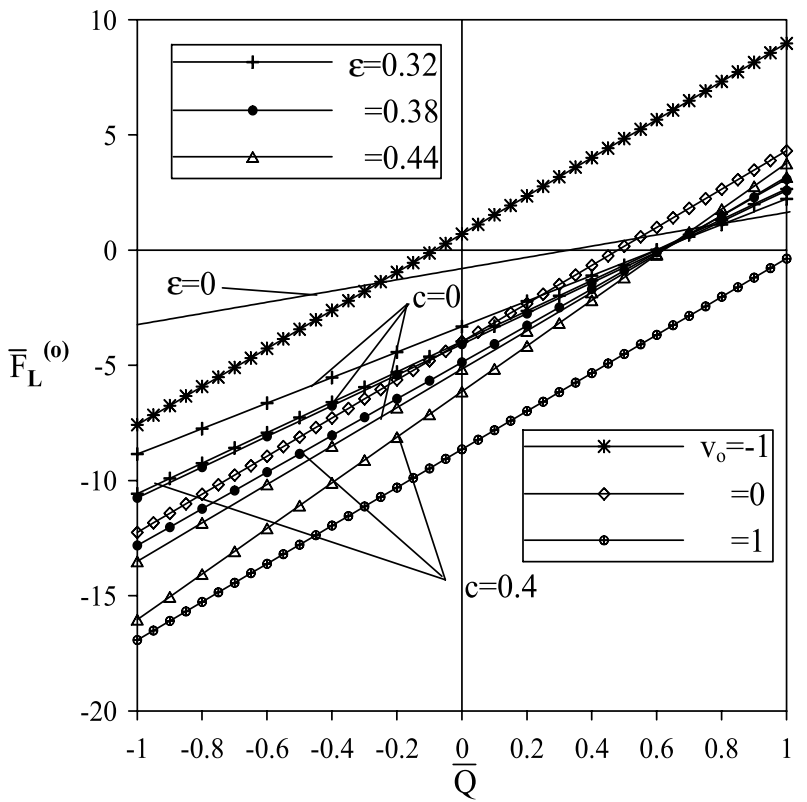

Figure 19. Outer friction force versus flow rate $\bar{Q}$ for a nonuniform annulus for different values of $\epsilon$ and $C$ at $\phi=0.3$ and $V_{0}=0.5$, and for different values of $V_{0}$ at $C=0, \phi=0.4$ and $\epsilon=0.38$.

\section{Conclusion}

The problem of peristaltic flow through a uniform and nonuniform annulus filled with an incompressible viscous and Newtonian fluid mixed with rigid spherical particles of identical size is investigated theoretically. The exact expressions for axial velocity of the fluid and particulate phase and axial pressure gradient are obtained analytically. Graphical results are presented for the pressure rise over the length of a uniform and non-uniform annulus and inner and outer friction force. The main findings can be summarised as follows:

(1) The pressure rise increases with the increase in the radius ratio, $\epsilon$, and particle concentration, $C$.

(2) The magnitude of the pressure rise for a nonuniform tube is much smaller than the corresponding value for a uniform tube at the same values of the parameters.

(3) The pressure rise increases with the increase in the value of the amplitude ratio, $\phi$.

(4) The pressure rise increases with the increase in $V_{0}$ (the inner tube moves in the direction of the peristaltic wave).

(5) The peristaltic pumping region becomes wider with the increase in the radius ratio, $\epsilon$, particle concentration, $C$ and $V_{0}$.

(6) The inner and outer friction forcs have the opposite behaviour compared with the pressure rise.
(7) The outer friction force is greater than the inner friction force at the same values of the parameters.

(8) In the limit when $r_{1} \rightarrow 0$ at $C=0$, our results of pressure rise and the outer friction force are the same as the results obtained by Gupta and Seshadri (1976).

\section{References}

[1] Ali N, Hayat T, Sajid M. 2007. Peristaltic flow of a couple stress fluid in an asymmetric channel. Biorheology. 44:125.

[2] Ali N. Hussain Q, Hayat T, Asghar S. 2008. Slip effects on the peristaltic transport of MHD fluid with variable viscosity. Phys. Lett. A. 372:1477.

[3] Brown TD, Hung TK. 1977. Computational and experimental investigations of two-dimensional nonlinear peristaltic flows. J Fluid Mech. 83:249.

[4] Charm SE, Kurland GS. 1974. Blood flow and microcirculation. New York: John Wiley.

[5] Cotton PB, Williams CB. 1990. Practical gastrointestinal endoscopy. Third edition. London: Oxford university press.

[6] Drew DA. 1979. Stability of a stokes layer of a dusty gas. Phys Fluids. 22:2081.

[7] El Shehawey EF, Mekheimer KS. Couple-stresses in peristaltic transport of fluids. J Phys D: Appl Phys. 27:1163.

[8] Elshehawey EF, El-Saman AE., El-Shahed M, Dagher M. 2005. Peristaltic transport of a compressible viscous liquid through a tapered pore. Appl. Math. Comput. 169:526.

[9] Eytan O, Jaffa JA, Elad D. 2001. Peristaltic flow in a tapered channel: Application to embryo transport within the uterine cavity. Med. Eng Phys. 23:473.

[10] Gupta BB, Seshadri V. 1976. Peristaltic pumping in nonuniform tubes. J Biomech. 9:105.

[11] Hakeem AE, Naby AE, El Misery AEM, Kareem MFAE. 2006. Effects of a magnetic field on trapping through peristaltic motion for generalized Newtonian fluid in channel. Phys A. 367:79.

[12] Hayat T, Ali N. 2006. Peristaltically induced motion of a MHD third grade fluid in a deformable tube. Phys A. 370:225.

[13] Hayat T, Ali N, Abbas Z. 2007. Peristaltic flow of a micropolar fluid in a channel with different wave forms. Phys Lett A. $370: 331$.

[14] Hayat T, Ali N, Asghar S. 2007a. An analysis of peristaltic transport for flow of a Jeffrey fluid. Acta Mech. 193:535.

[15] Hayat T, Ali N, Asghar S. 2007b. Hall effects on peristaltic flow of a Maxwell fluid in a porous medium. Phys Lett A. 363:397.

[16] Hayat T, Ali N, Asghar S. 2007c. Peristaltic motion of a Burger's fluid in a planar channel. Appl Math Comput. 186:309.

[17] Hayat T, Ali N, Asghar S, Siddiqui AM. 2006. Exact peristaltic flow in tubes with an endoscope, Appl Math Comput. $182: 359$

[18] Hayat T, Afsar A, Khan M, Asghar S. 2007. Peristaltic transport of a third order fluid under the effect of a magnetic field. Comput Math Appl. 53:1074.

[19] Hayat T, Mahomed FM, Asghar S. 2005. Peristaltic flow of a magnetohydrodynamic Johnson-Segalman fluid. Nonlinear Dyn. 40:375.

[20] Hayat T, Momoniat E, Mahomed FM. 2006. Endoscope effects on MHD peristaltic flow of a power-law fluid. Math Problems Eng. 2006:ID 84276. 
[21] Hayat T, Wang Y, Siddiqui AM, Hutter K, Asghar S. 2002. Peristaltic transport of a third order fluid in a circular cylindrical tube. Math Models Methods Appl Sci. 12:1691.

[22] Latham TW. 1966. Fluid motion in a peristaltic pump. M.Sc. Thesis. Cambridge, MA: MIT; 1966.

[23] Lee JS, Fung YC. 1971. Flow in non-uniform small blood vessels. Microvasc Res. 3:272.

[24] Mekheimer KS. 2002. Peristaltic transport of a couple-stress fluid in a uniform and non-uniform channels. Biorheology. 39:755.

[25] Mekheimer KS. 2003a. Non-linear peristaltic transport of magneto-hydrodynamic flow in an inclined planar channel. Arabian J Sci Eng. 28(2A):183.

[26] Mekheimer KS. 2003b. Non-linear peristaltic transport through a porous medium in an inclined planar channel. J Porous Medium. 6:189.

[27] Mekheimer KS. 2005. Peristaltic transport of a Newtonian fluid through a uniform and non-uniform annulus. Arabian J Sci. Eng. 30(1A):69.

[28] Mekheimer KS. 2008. Effect of the induced magnetic field on peristaltic flow of a couple stress fluid. Phys Lett A. 372:4271.

[29] Mekheimer KS, Abd elmaboud Y. 2008a. Peristaltic flow of a couple stress fluid in an annulus: Application of an endoscope. Phys A. 387:2403.

[30] Mekheimer KS, Abd elmaboud Y. 2008b. Peristaltic flow through a porous medium in an annulus: Application of an endoscope. Appl Math Information Sci. 2(1):103.

[31] Mekheimer KS, Abd elmaboud Y. 2008c. The influence of heat transfer and magnetic field on peristaltic transport of a Newtonian fluid in a vertical annulus: Application of an endoscope. Phys Lett A. 372:1657.

[32] Mekheimer KS, El Shehawey EF, Elaw AM. 1998. Peristaltic motion of a particle-fluid suspension in a planar channel. Int J Theor Phys. 37:2895.

[33] Misra CJ, Pandey KS. 1994. Peristaltic transport of a particle-fluid suspension in a cylindrical tube. Comput. Math Appl. 28:131.

[34] Misra CJ, Pandey SK. 1995. Peristaltic transport in a tapered tube. Math Comput Modell. 22:137.
[35] Ramachandra RA, Usha S. 1995. Peristaltic transport of two immiscible viscous fluids in a circular tube. J Fluid Mech. 298:271.

[36] Rathish Kumar BV, Naidu KB. 1995. A numerical study of peristaltic flows. Comput Fluids. 24:161.

[37] Shapiro AH, Jaffrin MY, Weinberg SL. 1969. Peristaltic pumping with long wavelengths at low Reynolds number. J Fluid Mech. 37:799.

[38] Srivastava VP, Saxena M. 1997. Particulate suspension flow induced by sinusoidal peristaltic waves. Jpn J Appl Phys. $36: 385$.

[39] Srivastava LM, Srivastava VP. 1988. Peristaltic transport of a Power-Law fluid: Application to the ductus efferentes of the reproductive tract. Rheol Acta. 27:428.

[40] Srivastava LM, Srivastava VP. 1989. Peristaltic transport of a particle-fluid suspension. J Biomech Eng. 111:157.

[41] Srivastava PV, Srivastava ML. 1997. Influence of wall elasticity and poiseuille flow on peristaltic induced flow of a particle-fluid mixture. Int J Eng Sci. 35:1359.

[42] Srivastava LM, Srivastava VP, Sinha SN. 1983. Peristaltic transport of a physiological fluid: Part I. Flow in non-uniform geometry. Biorheology. 20:153.

[43] Takabatake S, Ayukawa K. 1982. Numerical study of two dimensional peristaltic flows. J Fluid Mech. 122:439.

[44] Takabatake S, Ayukawa K, Mori A. 1988. Peristaltic pumping in circular cylindrical tubes: A numerical study of fluid transport and its efficiency. J Fluid Mech. 193:267.

[45] Tang D, Shen M. 1993. Non-stationary peristaltic and transport of a heat-conducting fluid. J Math Anal Appl. 174(1):265.

[46] Vajravelu K, Sreenadh S, Babu VR. 2005. Peristaltic transport of a Herschel-Bulkley fluid in an inclined tube. Int $\mathbf{J}$ Non-Linear Mech. 40:83.

[47] Wang Y, Hayat T, Ali N, Oberlack M. 2008. Magnetohydrodynamic peristaltic motion of a Sisko fluid in a symmetric or asymmetric channel. Phys A. 387:347.

[48] Wiedeman MP. 1963. Dimensions of blood vessels from distributing artery to collecting vein. Circ Res. 12:375.

[49] Zien TF, Ostrach SA. 1970. A long wave approximation to peristaltic motion. J Biomech. 3:63. 

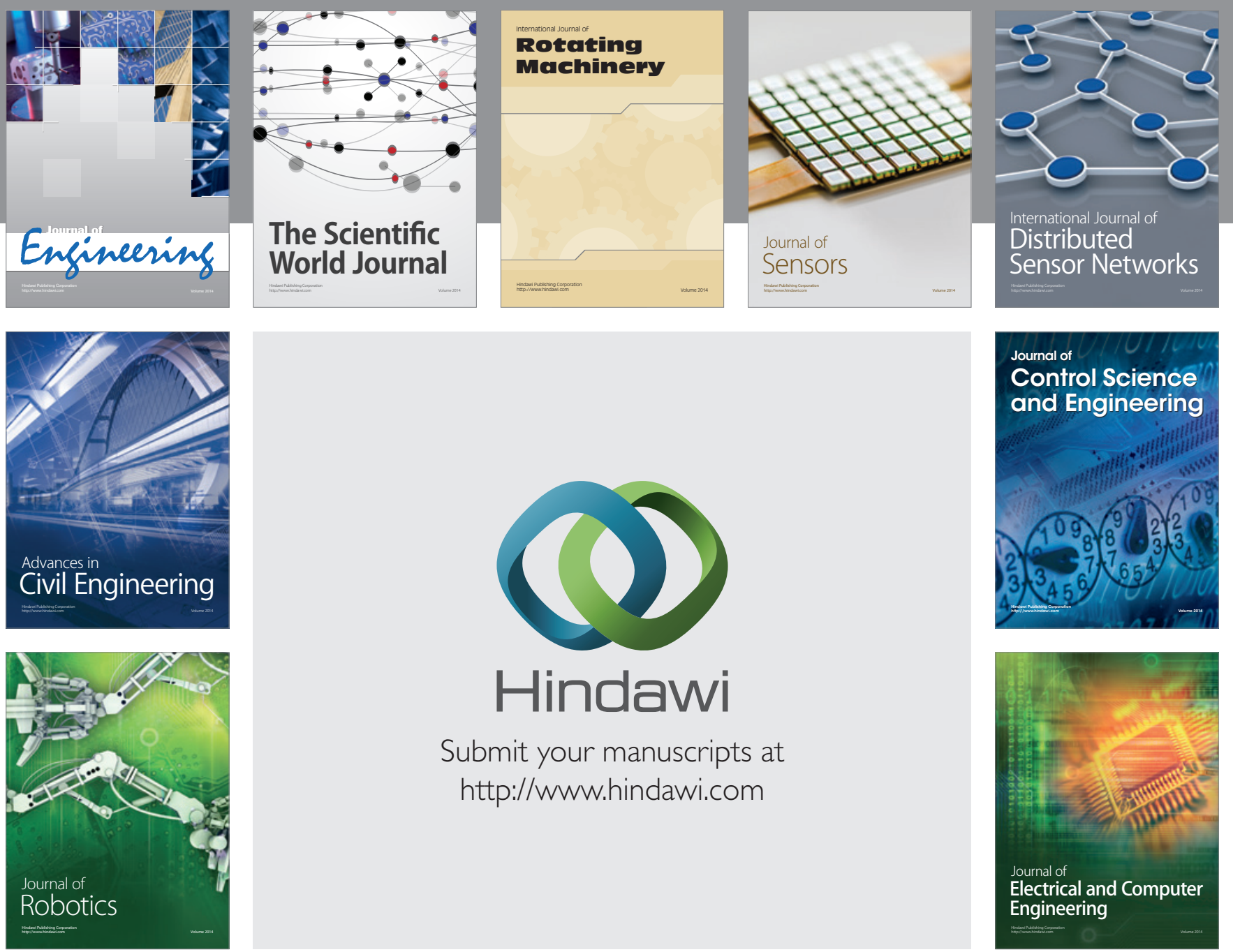

Submit your manuscripts at

http://www.hindawi.com
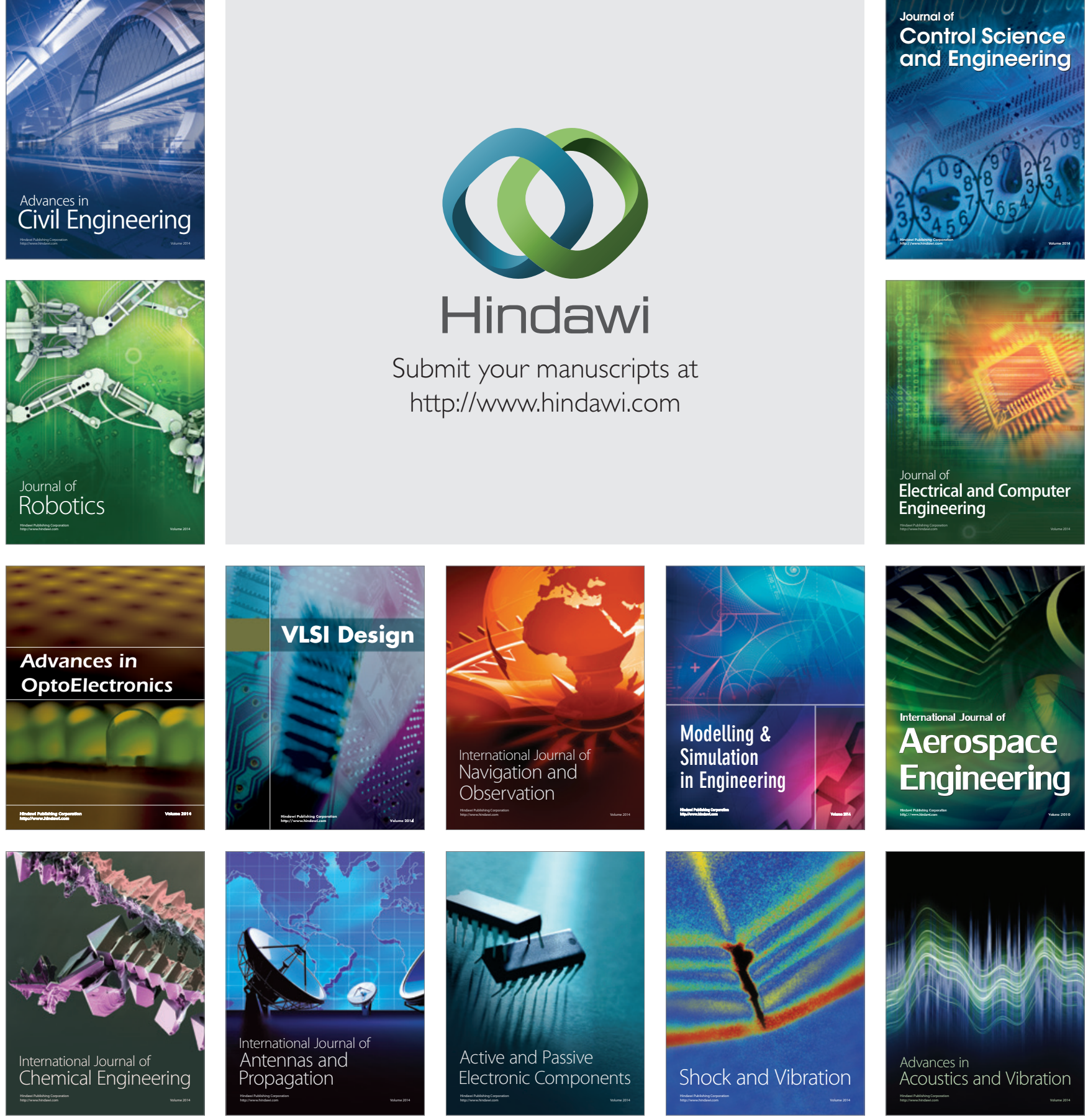